\title{
Modelos estatísticos para seleção de dosadores helicoidais com diferentes dispositivos de descarga de fertilizante
}

\author{
Cristian J. Franck ${ }^{1}$, Airton dos S. Alonçoํㅜ, Otávio D. da C. Machado ${ }^{2}$, \\ Tiago R. Francetto ${ }^{1}$, Dauto P. Carpes ${ }^{1} \&$ Mateus P. Bellé ${ }^{1}$
}

${ }^{1}$ Programa de Pós-Graduação em Engenharia Agrícola/Universidade Federal de Santa Maria. Santa Maria, RS. E-mail: cristian_franck@yahoo.com.br (Autor correspondente); airtonalonco@gmail.com; tiagofrancetto@gmail.com; dautocarpes@gmail.com; mateuspotrichbelle@yahoo.com.br

${ }^{2}$ Instituto Federal do Rio Grande do Sul. Bento Gonçalves, RS. E-mail: otaviomachado@hotmail.com

\section{Palavras-chave:}

semeadora adubadora

taxa de aplicação de fertilizantes mecanização agrícola

\begin{abstract}
R E S U M O
O presente trabalho teve por objetivo desenvolver uma ferramenta capaz de expressar matematicamente a taxa de aplicação de fertilizante em função do tipo de dosador, inclinação longitudinal, inclinação transversal e velocidade de acionamento do dosador. Foram utilizados três dosadores helicoidais com dispositivos de descarga por gravidade, transbordo transversal e transbordo lateral e adotado o delineamento composto central rotacional, com três variáveis independentes. A partir dos modelos ajustados constatou-se que os dispositivos de descarga apresentam diferentes implicações em relação aos efeitos de inclinações longitudinais e transversais de operação. O dosador com descarga por gravidade demonstrou maior sensibilidade quanto aos efeitos das inclinações de operação e o dosador com descarga por transbordo lateral demonstrou menor sensibilidade. A análise de regressão demonstrou que todos os modelos foram significativos, sendo encontrados baixos erros relativos e absolutos dos dados previstos pelos modelos.
\end{abstract}

\section{Key words:}

seed drill

fertilizer application rate

agricultural mechanization

\section{Mathematical models for selection of helical fertilizer metering mechanism with different fertilizer discharge}

\begin{abstract}
A B S T R A C T
This study aimed to develop a tool capable of expressing mathematically the rate of fertilizer application depending on the type of distributor, longitudinal slope, cross slope and driving speed of distributor. Three helical distributors with gravity discharge, transverse discharge through overflow and side discharge through overflow were used. The central composite rotational design with three independent variables was adopted. From the adjusted models it was found that the discharge devices have different implications for the effects of longitudinal and transversal slopes of operation. The distributor with gravity discharge showed greater sensitivity to the effects of slopes and the distributor side discharge through overflow showed lower sensitivity. Regression analysis showed that all models were significant, and found low relative and absolute errors of the data predicted by the models.
\end{abstract}




\section{INTRODUÇÃo}

Em se tratando da reposição de nutrientes em operação simultânea à semeadura, os componentes responsáveis pela dosagem de fertilizante são os mecanismos dosadores de fertilizante de semeadoras-adubadoras. Tais mecanismos podem assumir diversas disposições construtivas sendo encontradas, no mercado brasileiro, várias opções. Mesmo com os avanços tecnológicos dos mecanismos utilizados na aplicação de fertilizantes é possível afirmar que, por diversos motivos, a qualidade da distribuição ainda é aquém do desejado (Ferreira et al., 2010; Bonotto, 2012; Garcia et al., 2012).

Entre os principais fatores geradores de erros na aplicação de fertilizantes podem ser citados os efeitos de inclinações transversais e longitudinais de trabalho. Ferreira et al. (2010) constataram, ao analisar, em diferentes condições de trabalho, o desempenho de dois dosadores helicoidais, com descarga por transbordo transversal e por gravidade, que a inclinação longitudinal afeta a taxa de aplicação e que, independente do tipo do mecanismo dosador, da velocidade de acionamento e do passo do helicoide, a taxa de aplicação obteve maior percentual de variação para as maiores inclinações, em módulo, ou seja, negativas e positivas.

Bonotto (2012) avaliou o desempenho de dosadores em função de inclinações longitudinais e transversais, separadamente. Quanto às inclinações longitudinais, o autor afirma que houve efeito significativo sobre a taxa de aplicação para os cinco mecanismos dosadores estudados, com os três tipos de fertilizante sendo observada tendência de aumento da taxa de aplicação quando operando em inclinações positivas e redução na taxa de aplicação para inclinações negativas. Quanto às inclinações transversais, este autor observou que também ocorreu efeito significativo porém não foi possível observar tendências no comportamento pois para cada vazão, fertilizante e dosador, houve comportamentos diferentes. $\mathrm{O}$ coeficiente de variação médio para inclinações transversais foi inferior a $5 \%$, sendo o menor valor observado para o dosador do tipo rotor helicoidal com descarga por transbordo transversal.

Dentre os tipos de dosadores se destacam os helicoidais, os quais fazem uso do mesmo princípio de solução para o transporte de materiais das roscas transportadoras, sendo assim pertinente fazer uma analogia entre os mesmos. Shimizu \& Cundall (2001) citam que esses tipos de mecanismos apresentam, como principais vantagens, seu design compacto, pouca manutenção e capacidade de transportar materiais continuamente e de forma segura em relação aos quais Owen \& Cleary (2009) afirmam que inclinações longitudinais podem reduzir o fluxo de produto em até $47 \%$.

Segundo Grisso et al. (2011) o aumento da eficácia da aplicação de fertilizantes está diretamente relacionado ao desenvolvimento de tecnologias capazes de aplicar a quantidade necessária em cada local da lavoura reduzindo erros ocasionados por diferentes fatores. A agricultura de precisão $(\mathrm{AP})$, principalmente através da tecnologia de aplicação a taxas variáveis, é uma das tecnologias que visam ao aumento da eficiência, redução de custos e de impactos ambientais. A taxa variável pode ser baseada em dois sistemas de prescrição: por mapas de aplicação georreferenciados ou por cálculos em tempo real por algoritmos abastecidos por dados de sensores (Yuan et al., 2010).

Para utilização de taxa variável de fertilizantes durante o processo de implantação de culturas a semeadora-adubadora deverá ser adequada previamente. Para isto é necessário um sistema de controle eletrônico, responsável por definir a posição geográfica da máquina, associar esta posição a uma taxa de aplicação oriunda de um mapa ou de sensores e comandar o acionamento dos dosadores de forma a atingir a quantidade desejada. Além disto, deve ser calibrado para haver uma correspondência entre a prescrição e a dosagem real (Rossato et al., 2012).

Os controladores eletrônicos para taxa variável são definidos por Umezu \& Cappelli (2006) como dispositivos eletrônicos, normalmente microprocessados que têm a função de variar regulagens do equipamento para alterar a taxa de aplicação com base em informações obtidas dos sensores elou de um mapa de aplicação. Para adequação da taxa de aplicação de fertilizantes o controlador executa uma rotina de cálculos baseada em algoritmos os quais, segundo Lopes (2010) são instrumentos de programação computacional que definem uma sequência passo a passo de tarefas ou atividades que devem ser realizadas para resolução de cálculos, leitura de sinais de sensores, entre outras atividades; no entanto, não são reconhecidas, atualmente, instalações de complementos nos sistemas que realizem a leitura da inclinação e, portanto, adequação da sua dose.

Com o intuito de sanar esta deficiência é necessário o estabelecimento de novas rotinas de cálculo para o desenvolvimento de futuros algoritmos, por modelos estatísticos ou matemáticos, que considerem os efeitos das inclinações. Garcia (2007) afirma que a modelagem é uma ferramenta potente para auxiliar no projeto dos sistemas de controle para aplicação a taxas variáveis. Olieslagers et al. (1996) afirmam que para o desenvolvimento desses sistemas de controle é conveniente desenvolver modelos que considerem parâmetros relativos ao fertilizante bem como as disposições construtivas e operacionais dos dosadores utilizados.

O objetivo do presente trabalho foi desenvolver, através do delineamento composto central rotacional, uma ferramenta capaz de expressar matematicamente a taxa de aplicação de um fertilizante em função do tipo de dosador, inclinação longitudinal de trabalho, inclinação transversal de trabalho e velocidade de acionamento que possa ser utilizada como recurso para o desenvolvimento de sistemas eletrônicos de controle da taxa de aplicação de fertilizantes de semeadorasadubadoras e escolha de dosadores em função das condições de operação às quais estes serão submetidos.

\section{Material e Métodos}

O trabalho foi conduzido nas instalações do Laboratório de Pesquisa e Desenvolvimento de Máquinas Agrícolas (LASERG), vinculado à Universidade Federal de Santa Maria, Rio Grande do Sul, Brasil. Foi utilizada a bancada para testes com dosadores de fertilizantes de semeadoras-adubadoras em linhas, BANFERTI II, sendo cada dosador acionado individualmente. 
Foram utilizados três dosadores de fertilizante do tipo helicoidal com diferentes dispositivos de descarga. $\mathrm{O}$ dosador D1 possuía dispositivo cuja descarga se dá por transbordo transversal, que consiste em uma barreira à passagem do fertilizante, posicionada ao fim do helicoide, perpendicularmente ao eixo do mesmo. O dosador D2 possuía descarga por transbordo lateral, caso em que a barreira se encontra, ao final do helicoide, posicionada paralelamente ao eixo do mesmo. O dosador D3 possuía descarga por gravidade, ou seja, nenhuma barreira impede a descarga do fertilizante o qual é liberado ao fim do helicoide, na posição inferior. Para todos os dosadores o helicoide utilizado foi o fornecido por seu respectivo fabricante, com passo e diâmetro de 50,8 mm sendo acionados no sentido horário.

O fertilizante utilizado foi do tipo mistura de grânulos com formulação, declarada pelo fabricante, N-P-K 05-20-20. Após análise das propriedades físicas do fertilizante constatou-se que o mesmo possuía densidade de $980 \mathrm{~kg} \mathrm{~m}^{-3}$, ângulo de repouso de $31,78^{\circ}$ e teor de água de $4,12 \%$ para base seca. A análise granulométrica foi realizada de acordo com ABNT (2003) e o percentual retido acumulado nas peneiras com perfurações de 4, 2, 1 e 0,5 mm foi de 2,28, 78,21, 99,31 e 99,97\% respectivamente.

$\mathrm{O}$ acionamento do eixo dos dosadores foi realizado por meio de um motor elétrico de $0,735 \mathrm{~kW}$. O controle das velocidades de acionamento foi realizado através de inversor de frequências marca WEG, modelo CFW 10. A configuração das inclinações foi realizada manualmente, sendo conferida com clinômetro digital marca Pittsburgh, modelo 95998. Seguindo a metodologia proposta por Bonotto (2012) para cada dosador foi utilizado um reservatório cúbico confeccionado em madeira e com visor em vidro, com capacidade para $0,0188 \mathrm{~m}^{3}$, sendo preenchido durante todos os testes em $50 \%$ de seu volume. Cada observação foi composta de um tempo aproximado de estabilização de $15 \mathrm{~s}$ após a configuração da frequência de acionamento correspondente, seguido da efetiva coleta da quantidade de fertilizante distribuída pelos dosadores em $30 \mathrm{~s}$. Foi utilizada balança eletrônica com precisão de $1 \mathrm{~g}$ e os dados foram processados para representar a vazão correspondente para unidade $\mathrm{g} \mathrm{min}^{-1}$.

A realização dos experimentos foi baseada no delineamento composto central rotacional (DCCR), com três variáveis independentes, inclinação longitudinal (IL), inclinação transversal (IT) e velocidade de acionamento (R) e uma variável dependente, taxa de aplicação (Y). A composição do delineamento foi baseada segundo Dacanal \& Menegalli (2010), sendo este composto por um fatorial $2^{3}+6$ pontos axiais +5 repetições no ponto central. Segundo Rodrigues \& Iemma (2009) o DCCR utiliza combinações específicas das variáveis independentes a fim de que sejam explorados os seus efeitos com menos unidades experimentais (UE); para tanto, os tratamentos formaram um contraste ortogonal e as variáveis foram utilizadas na forma de códigos, sendo; $-1,68$; $-1 ; 0 ; 1 ; 1,68$.

O fatorial $2^{3}$ corresponde a dois níveis de cada uma das três variáveis independentes, identificados pelos códigos -1 e 1, totalizando 8 UE. Os pontos axiais correspondem aos valores extremos das variáveis independentes identificados pelos códigos -1,68 e 1,68 totalizando 6 UE. As repetições no ponto central, identificadas pelo código 0 , são utilizadas para determinação do erro puro.

Para cada uma das variáveis independentes foram encontrados, na literatura, valores extremos já estudados, correspondendo aos códigos -1,68 e 1,68. Através de interpolação foram então determinados os valores correspondentes aos códigos $-1,0$ e 1 . Para IL e IT os valores extremos de $-11^{\circ} \mathrm{e}$ $11^{\circ}$, foram definidos de acordo com adaptação do estabelecido por ABNT (1994). Para R, os valores extremos de 25 e 125 rpm foram definidos em acordo com a faixa utilizada por Camacho-Tamayo et al. (2009), Ferreira et al. (2010), Garcia (2011) e Bonotto (2012). Na Tabela 1 podem ser observados os valores absolutos correspondentes aos códigos para cada uma das variáveis independentes.

Tabela 1. Valores em código e absolutos utilizados nos experimentos

\begin{tabular}{cccccc}
\hline $\begin{array}{c}\text { Valores em código } \\
\text { (adimensionais) }\end{array}$ & $\mathbf{- 1 , 6 8}$ & $-\mathbf{1}$ & $\mathbf{0}$ & $\mathbf{1}$ & $\mathbf{1 , 6 8}$ \\
IL ( $\left.{ }^{\circ}\right)$ & -11 & 6,55 & 0 & 6,55 & 11 \\
IT ( $\left.{ }^{\circ}\right)$ & -11 & 6,55 & 0 & 6,55 & 11 \\
R (rpm) & 25 & 45 & 75 & 105 & 125 \\
\hline
\end{tabular}

Foram ajustados, para cada dosador, os estimadores dos parâmetros do modelo estatístico proposto, Eq. 1, através do software Statistica 7.0. O modelo proposto considera o efeito de uma constante, efeito linear e quadrático de cada uma das variáveis independentes, além do efeito da interação entre elas.

$$
\begin{aligned}
\hat{\mathrm{Y}} & =\mathrm{b}_{1}+\mathrm{b}_{2} \mathrm{IL}+\mathrm{b}_{3} \mathrm{IL}^{2}+\mathrm{b}_{4} \mathrm{IT}+\mathrm{b}_{5} \mathrm{IT}^{2}+ \\
& +\mathrm{b}_{6} \mathrm{R}+\mathrm{b}_{7} \mathrm{R}^{2}+\mathrm{b}_{8} \mathrm{ILIT}+\mathrm{b}_{9} \mathrm{ILR}+\mathrm{b}_{10} \mathrm{ITR}
\end{aligned}
$$

em que:

Y - taxa de aplicação de fertilizantes, $\mathrm{g} \mathrm{min}^{-1}$

$b_{1}, b_{2}, b_{3}, \ldots, b_{10}$ - estimadores dos parâmetros do modelo, adimensionais

IL - inclinação longitudinal, em código adimensional

IT - inclinação transversal, em código adimensional

$\mathrm{R}$ - velocidade de acionamento do dosador, em código adimensional

Para facilitar o entendimento os estimadores foram denominados a partir da variável através da qual são multiplicados. Por exemplo, os estimadores $b_{2}$ e $b_{3}$, que são multiplicados pelas variáveis Inclinação Longitudinal (IL) e Inclinação Longitudinal elevada ao quadrado $\left(\mathrm{IL}^{2}\right)$, são denominados IL e $\mathrm{IL}^{2}$, respectivamente; assim e durante a discussão dos efeitos de cada variável não se faz necessário mencionar o estimador nem a variável a que este está ligado.

É importante salientar que para inclinações longitudinais, valores positivos (Figura 1A) e negativos (Figura 1B) simulam o deslocamento da semeadora em trajetória ascendente e descendente, respectivamente. Para inclinações transversais valores negativos (Figura 1C) e positivos (Figura 1D) simulam inclinações para a direita e a esquerda, respectivamente, considerando o observador localizado na parte posterior dos dosadores.

O modelo estatístico proposto possui dez estimadores porém alguns desses podem não ser significativos. Para 
A.

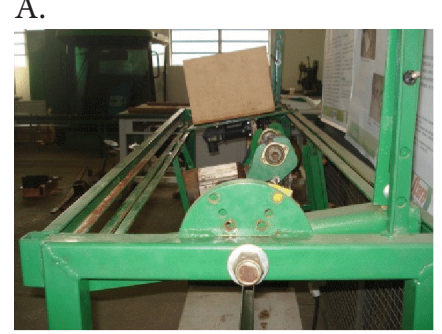

C.

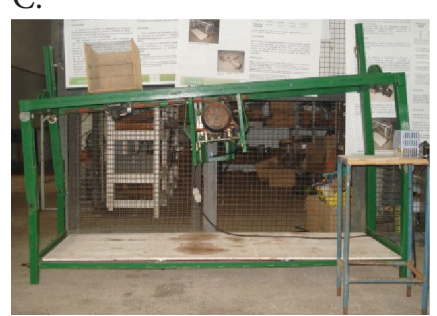

Figura 1. Inclinações da BANFERTI II: Inclinação longitudinal positiva (A), Inclinação longitudinal negativa (B), Inclinação transversal negativa (C), Inclinação transversal positiva (D)

determinar os estimadores significativos e eliminar do modelo os estimadores não significativos foi realizada análise de variância sendo este processo denominado reparametrização.

Como os tratamentos são em código, os modelos também o são. Para que sejam inseridos no modelo os valores absolutos das variáveis é oportuno realizar a decodificação do modelo. Como os tratamentos foram expressos como matrizes é possível aplicar, sobre elas, suas propriedades; logo, fazendo uso da propriedade da matriz inversa, conforme a Eq. 2, realizou-se a decodificação dos modelos.

$$
b=\operatorname{inv}\left(\operatorname{trat}^{\prime} \times \operatorname{trat}\right) \times \operatorname{trat}^{\prime} \times y
$$

em que:

b - matriz dos estimadores do modelo

inv - matriz inversa

trat - matriz dos tratamentos

trat' - matriz transposta dos tratamentos

y - matriz dos resultados de predição do modelo codificado

Para comprovar a significância dos modelos de maneira semelhante a Dacanal \& Menegalli (2010) realizou-se a análise de regressão através de análise de variância e teste $\mathrm{F}$. Também foram determinados o coeficiente de determinação $\mathrm{R}^{2}$, os erros de ajuste e relativos. A determinação desses últimos foi realizada por meio das Eqs. 3 e 4, respectivamente, para o que foram inserido, nos modelos, os mesmos valores de inclinações longitudinais, transversais e velocidade de acionamento utilizados em cada UE nas coletas de dados, sendo então comparados os valores previstos pelo modelo com os valores observados.

A comprovação da qualidade do modelo se deu também através da análise do número de variáveis significativas, sua magnitude e do seu sinal (positivo ou negativo) ou seja, modelos com maior número de variáveis significativas e com maior magnitude são mais sensíveis ao efeito das inclinações de operação. Para comprovar tal comportamento foi realizada uma analogia entre os modelos e os coeficientes de variação determinados em pesquisas com condições experimentais semelhantes nos trabalhos de Bonotto (2012) e Ferreira et al. (2010).

$$
\mathrm{EA}=\mathrm{VO}-\mathrm{VP}
$$

em que:

EA - erro de ajuste, $\mathrm{g} \mathrm{min}^{-1}$

VO - valor observado, $\mathrm{g} \mathrm{min}^{-1}$

VP - valor previsto pelo modelo, $\mathrm{g} \mathrm{min}^{-1}$

$$
\mathrm{ER}=\left|\frac{(\mathrm{VO}-\mathrm{VP})}{\mathrm{VO}} \times 100\right|
$$

em que:

ER - erro relativo, \% em módulo

VO - valor observado, $\mathrm{g} \mathrm{min}^{-1}$

VP - valor previsto pelo modelo, $\mathrm{g} \mathrm{min}^{-1}$.

\section{Resultados e Discussão}

Os modelos estatísticos desenvolvidos demonstram que os dosadores influenciam de forma diferente a distribuição de fertilizantes pelo efeito de inclinações de trabalho, já que cada modelo apresentou diferentes valores para os estimadores dos parâmetros. Para todos os dosadores foi observado efeito significativo dos estimadores ligados ao efeito linear de IL. Em nenhum dos dosadores foi observado o efeito quadrático tanto de $\mathrm{IL}^{2}$ quanto de $\mathrm{IT}^{2}$. $\mathrm{O}$ efeito linear de IT foi significativo apenas para os dosadores D1 e D3 e o efeito da interação entre IL e IT foi significativo apenas para D2 enquanto a interação entre IL e R foi significativa para todos.

$\mathrm{Na}$ Tabela 2 estão apresentados os tratamentos e os valores utilizados para a determinação dos estimadores dos parâmetros do modelo matemático de cada dosador. É possível observar que as vazões foram ligeiramente diferentes sendo que, em média, D2 apresentou a menor vazão

Tabela 2. Tratamentos em código e valores observados nas coletas de dados

\begin{tabular}{cccccc}
\hline \multicolumn{3}{c}{ Tratamentos } & & \multicolumn{3}{c}{ Valores observados $\left(\mathbf{g ~ m i n}^{-1}\right)$} \\
\hline IL & IT & R & D1 & D2 & D3 \\
-1 & -1 & -1 & $1.830,00$ & 844,00 & 1700,00 \\
1 & -1 & -1 & $2.140,00$ & 942,00 & 2098,00 \\
-1 & 1 & -1 & $1.916,00$ & 796,00 & 1654,00 \\
1 & 1 & -1 & $2.158,00$ & 986,00 & 1980,00 \\
-1 & -1 & 1 & $4.408,00$ & 2002,00 & 3934,00 \\
1 & -1 & 1 & $4.918,00$ & 2322,00 & 4524,00 \\
-1 & 1 & 1 & $4.516,00$ & 1972,00 & 3830,00 \\
1 & 1 & 1 & $4.928,00$ & 2360,00 & 4422,00 \\
$-1,68$ & 0 & 0 & $2.968,00$ & 1324,00 & 2604,00 \\
1,68 & 0 & 0 & $3.606,00$ & 1656,00 & 3374,00 \\
0 & $-1,68$ & 0 & $3.288,00$ & 1550,00 & 3004,00 \\
0 & 1,68 & 0 & $3.400,00$ & 1484,00 & 2868,00 \\
0 & 0 & $-1,68$ & $1.094,00$ & 460,00 & 1044,00 \\
0 & 0 & 1,68 & $5.570,00$ & 2618,00 & 4936,00 \\
0 & 0 & 0 & $3.356,00$ & 1496,00 & 2986,00 \\
0 & 0 & 0 & $3.314,00$ & 1520,00 & 2994,00 \\
0 & 0 & 0 & $3.324,00$ & 1498,00 & 2978,00 \\
0 & 0 & 0 & $3.378,00$ & 1526,00 & 2960,00 \\
0 & 0 & 0 & $3.342,00$ & 1530,00 & 2956,00 \\
\hline & Média & & $3.339,68$ & $1.520,32$ & $2.991,89$ \\
\hline
\end{tabular}


comprovando que apesar de todos os dosadores utilizarem helicoide com mesmo passo e diâmetro, além das mesmas condições experimentais, o tipo de descarga, folgas entre carcaça e helicoide, rugosidade do helicoide e da carcaça, além de outras características construtivas do dosador, também afetam sua vazão.

$\mathrm{Na}$ Tabela 3 podem ser visualizados os estimadores dos parâmetros dos modelos estatísticos para os três dosadores estudados os quais já foram reparametrizados e decodificados. Todos os dosadores apresentaram 5 estimadores significativos entre os 10 possíveis. Para D1 e D3 os significativos foram os mesmos (Cte, IL, IT, R e ILR), mas com magnitudes diferentes denotando que apesar de ter os mesmos estimadores significativos, como sua magnitude é diferente, a influência das inclinações e sua velocidade de acionamento serão diferentes. Quanto ao efeito linear de IL, o dosador D2, com descarga por transbordo lateral, mostrou-se menos sensível ao seu efeito, apresentando estimador com menor valor fato este confirmado por Bonotto (2012), que encontrou o menor coeficiente de variação médio para este. Em relação ao desempenho dos dosadores D1 e D3, Ferreira et al. (2010) afirmam que o melhor desempenho foi observado para D1, com coeficientes de variação semelhantes ao observado para este dosador, por Bonotto (2012).

Para o efeito de IT é possível afirmar que o dosador menos sensível foi D2, já que não apresentou efeito significativo, ou seja, a operação em inclinações transversais de -11 a $11^{\circ}$ não alterará a taxa de aplicação. Entre D1 e D3, que apresentaram efeito significativo, o maior valor foi observado para D3. Já Bonotto (2012), encontrou o maior coeficiente de variação para D2 e valores próximos para D1 e D3.

Tanto Ferreira et al. (2010) quanto Bonotto (2012) afirmam que inclinações longitudinais positivas e negativas ocasionam aumentos e reduções, respectivamente, na taxa de aplicação. Este efeito foi observado para D1 e D3, que apresentaram valores positivos para o estimador IL; para D2, com estimador negativo, foi observado o efeito contrário. Quanto à inclinação transversal, D3, com estimador IT negativo, inclinações transversais negativas geram aumentos na taxa de aplicação enquanto inclinações transversais positivas geram reduções, de forma contrária ao observado para D1; já Bonotto (2012) não encontrou tendência de comportamento quanto aos efeitos dos sinais de inclinações transversais.

Tanto em relação a IL quanto a IT, o dosador D3 apresentou os maiores estimadores evidenciando que este possui maior sensibilidade devido, provavelmente, à ausência de dispositivo de descarga, concordando com Ferreira et al. (2010) que, em relação a IL apenas, observou o pior desempenho para dosadores com descarga por gravidade. Apenas o dosador D2 apresentou efeito significativo da interação entre IL e IT; todos os dosadores apresentaram efeito da interação entre IL e R, demonstrando que acréscimos da velocidade de acionamento aumentam o efeito de IL, de forma oposta ao observado por Owen \& Cleary (2009), Ferreira et al. (2010) e Bonotto (2012).

A partir da análise de regressão é possível constatar que todos os modelos foram significativos, $\rho$-valor menor que 0,05 ; além disto, os modelos apresentaram coeficientes de determinação, $\mathrm{R}^{2}$, superiores a 0,90 , sendo que os valores encontrados para os dosadores D1, D2 e D3 foram 0,9994; 0,9986 e 0,9988 , respectivamente; no entanto, além de significativos para comprovar a qualidade dos modelos é necessário que estes apresentem erros baixos, principalmente erros relativos. Analisando os resultados observados por Ferreira et al. (2010) e Bonotto (2012), é possível constatar que os erros na taxa de aplicação ocasionados pelo efeito de IL e IT são da ordem de $\pm 10 \%$. logo, para que os modelos sejam representativos permitindo sua utilização para a realização de simulações ou como ferramenta para correção da taxa de aplicação, é necessário que seus erros relativos médios sejam inferiores a $10 \%$. Caso os erros relativos médios sejam superiores a este limite o erro ocasionado pelo modelo será maior do que o erro ocasionado pelo efeito das inclinações dispensando o seu uso.

$\mathrm{Na}$ Tabela 4 são apresentados os erros absolutos e relativos. Para a determinação dos erros relativos foram considerados os valores em módulo a fim de que seja obtido o desvio percentual, independentemente se este foi para mais ou menos em relação ao valor observado na coleta de dados. Para todos os dosadores estudados, tanto os valores individuais de cada UE como os valores médios, os erros relativos foram inferiores ao limite de $10 \%$. Os valores dos erros bem como as UE, nos quais os maiores valores foram observados, apresentaram diferentes tendências para cada dosador. Observa-se, porém, que os maiores valores ficaram distribuídos entre as UE 9 a 14, nas quais foram estudados os valores extremos de cada variável, valores em código de $-1,68$ e 1,68; este fato pode apontar fragilidade tanto dos modelos estatísticos para predição em valores extremos como deficiência da metodologia do DCCR.

Devido às propriedades físicas do fertilizante, tais como densidade, ângulo de repouso, umidade e seu coeficiente de atrito, que segundo Mossmann et al. (2002) estão relacionadas à granulometria e ao contato entre partículas, tanto entre as frações do fertilizante, como entre fertilizante e dosado; logo, o tipo de fertilizante e suas propriedades físicas afetam as características da sua taxa de aplicação não permitindo a utilização de um modelo geral para um tipo de dosador, o que torna necessário o desenvolvimento de modelos para cada tipo de dosador e fertilizante.

Tabela 3. Estimadores dos parâmetros dos modelos para os três dosadores estudados

\begin{tabular}{cccccccccccc}
\hline Dosador & Cte. & IL & IL $^{2}$ & IT & IT $^{2}$ & $\mathbf{R}$ & $\mathbf{R}^{2}$ & ILIT & ILR & ITR \\
D1 & $-28,33$ & 10,69 & ns $^{*}$ & 4,59 & ns & 44,93 & ns & ns & 0,24 & ns \\
D2 & $-95,82$ & $-2,81$ & ns & ns & ns & 21,46 & $n s$ & 0,47 & 0,27 & ns \\
D3 & 51,74 & 13,78 & ns & $-6,70$ & ns & 38,95 & ns & ns & 0,29 & ns \\
\hline
\end{tabular}

* ns - Não significativo; Cte. - Constante; IL - Inclinação longitudinal linear; IL² - Inclinação longitudinal quadrática; IT - Inclinação transversal linear; IT² - Inclinação transversal quadrática; $\mathrm{R}$ - Velocidade de acionamento linear; $\mathrm{R}^{2}$ - Velocidade de acionamento quadrática; ILIT - Interação linear entre inclinações; ILR - Interação linear entre inclinação longitudinal e velocidade de acionamento; ITR - Interação linear entre inclinação transversal e velocidade de acionamento 
Tabela 4. Valores previstos pelos modelos e seus erros absolutos (EA) e seus erros relativos (ER) para os dosadores D1, D2 e D3

\begin{tabular}{|c|c|c|c|c|c|c|c|c|c|}
\hline \multirow[b]{2}{*}{ U.E. } & \multicolumn{3}{|c|}{ D1 } & \multicolumn{3}{|c|}{ D2 } & \multicolumn{3}{|c|}{ D3 } \\
\hline & $\begin{array}{l}\text { Valor previsto } \\
\qquad\left(\mathrm{g} \mathrm{min}^{-1}\right)\end{array}$ & $\begin{array}{c}\text { EA } \\
\left(g^{\mathrm{min}^{-1}}\right)\end{array}$ & $\begin{array}{l}\text { ER } \\
(\%)\end{array}$ & $\begin{array}{l}\text { Valor previsto } \\
\quad\left(\mathrm{g} \mathrm{min}^{-1}\right)\end{array}$ & $\begin{array}{c}\text { EA } \\
\left(g^{\mathrm{min}^{-1}}\right)\end{array}$ & $\begin{array}{l}\text { ER } \\
\text { (\%) }\end{array}$ & $\begin{array}{l}\text { Valor previsto } \\
\quad\left(\mathrm{g} \mathrm{min}^{-1}\right)\end{array}$ & $\begin{array}{c}\text { EA } \\
\left(g^{\mathrm{min}^{-1}}\right)\end{array}$ & $\begin{array}{l}\text { ER } \\
(\%)\end{array}$ \\
\hline 1 & $1.833,93$ & $-3,93$ & 0,21 & 833,53 & 10,47 & 1,24 & $1.680,40$ & 19,60 & 1,15 \\
\hline 2 & $2.114,59$ & 25,41 & 1,19 & 916,28 & 25,72 & 2,73 & $2.034,89$ & 63,11 & 3,01 \\
\hline 3 & $1.894,05$ & 21,95 & 1,15 & 793,53 & 2,47 & 0,31 & $1.592,68$ & 61,32 & 3,71 \\
\hline 4 & $2.174,71$ & $-16,71$ & 0,77 & 956,28 & 29,72 & 3,01 & $1.947,16$ & 32,84 & 1,66 \\
\hline 5 & $4.415,81$ & $-7,81$ & 0,18 & $2.005,72$ & $-3,72$ & 0,19 & $3.884,23$ & 49,77 & 1,27 \\
\hline 6 & $4.881,47$ & 36,53 & 0,74 & $2.298,46$ & 23,54 & 1,01 & $4.467,71$ & 56,29 & 1,24 \\
\hline 7 & $4.475,93$ & 40,07 & 0,89 & $1.965,72$ & 6,28 & 0,32 & $3.796,51$ & 33,49 & 0,87 \\
\hline 8 & $4.941,59$ & $-13,59$ & 0,28 & $2.338,46$ & 21,54 & 0,91 & $4.379,99$ & 42,01 & 0,95 \\
\hline 9 & $3.028,06$ & $-60,06$ & 2,02 & $1.322,19$ & 1,81 & 0,14 & $2.579,00$ & 25,00 & 0,96 \\
\hline 10 & $3.654,97$ & $-48,97$ & 1,36 & $1.704,80$ & $-48,80$ & 2,95 & $3.366,89$ & 7,11 & 0,21 \\
\hline 11 & $3.291,01$ & $-3,01$ & 0,09 & $1.513,50$ & 36,50 & 2,36 & $3.046,63$ & $-42,63$ & 1,42 \\
\hline 12 & $3.392,01$ & 7,99 & 0,23 & $1.513,50$ & $-29,50$ & 1,99 & $2.899,26$ & $-31,26$ & 1,09 \\
\hline 13 & $1.095,04$ & $-1,04$ & 0,09 & 440,66 & 19,34 & 4,20 & $1.025,55$ & 18,45 & 1,77 \\
\hline 14 & $5.587,99$ & $-17,99$ & 0,32 & $2.586,33$ & 31,67 & 1,21 & $4.920,34$ & 15,66 & 0,32 \\
\hline 15 & $3.341,51$ & 14,49 & 0,43 & $1.513,50$ & $-17,50$ & 1,17 & $2.972,95$ & 13,05 & 0,44 \\
\hline 16 & $3.341,51$ & $-27,51$ & 0,83 & $1.513,50$ & 6,50 & 0,43 & $2.972,95$ & 21,05 & 0,70 \\
\hline 17 & $3.341,51$ & $-17,51$ & 0,53 & $1.513,50$ & $-15,50$ & 1,03 & $2.972,95$ & 5,05 & 0,17 \\
\hline 18 & $3.341,51$ & 36,49 & 1,08 & $1.513,50$ & 12,50 & 0,82 & $2.972,95$ & 12,95 & 0,44 \\
\hline 19 & $3.341,51$ & 0,49 & 0,01 & $1.513,50$ & 16,50 & 1,08 & $2.972,95$ & $-16,95$ & 0,57 \\
\hline Média & $3.341,51$ & 21,13 & 0,65 & $1.513,50$ & 18,93 & 1,43 & $2.972,95$ & 29,87 & 1,16 \\
\hline
\end{tabular}

D1- Descarga por transbordo transversal, D2 - Descarga por transbordo lateral, D3 - Descarga por gravidade

\section{CONClusões}

1. Os modelos desenvolvidos foram, de acordo com os resultados obtidos, capazes de expressar a taxa de aplicação em função dos fatores estudados apresentando diferenças em função do tipo de mecanismo dosador demonstrando qualidade elevada e adequação para serem utilizados em simulações.

2. Nas condições experimentais estudadas as disposições construtivas dos dosadores utilizados, principalmente os dispositivos de descarga, apresentam diferentes efeitos em relação às inclinações longitudinais e transversais de operação.

3. O modelo desenvolvido para D1 (descarga por transbordo transversal) foi o que apresentou maior qualidade devido ao seu coeficiente de determinação mais elevado. $\mathrm{O}$ desempenho dos dosadores D2 (descarga por transbordo lateral) e D3 (descarga por gravidade), apesar de inferior, apresentou pequena diferença em relação a D1.

4. O dosador D3 mostrou-se mais sensível aos efeitos das inclinações longitudinais e transversais de operação.

\section{Literatura Citada}

ABNT - Associação Brasileira de Normas Técnicas. Projeto de norma 04: 015:06-004/1994 - Semeadora de precisão - ensaio de laboratório: método de ensaio. Rio de Janeiro: ABNT, 1994. 26p.

ABNT - Associação Brasileira de Normas Técnicas. NBR NM 248: Agregados - Determinação da composição granulométrica. Rio de Janeiro: ABNT, 2003. 6p.

Bonotto, G. J. Desempenho de dosadores de fertilizantes de semeadoras-adubadoras em linhas. Santa Maria: UFSM, 2012. 97p. Dissertação Mestrado

Camacho-Tamayo, J. H.; Barbosa, A. M.; Pérez, N. M.; Leiva, F. R.; Rodríguez, G. A. Operational characteristics of four metering systems for agricultural fertilizers and amendments. Engenharia Agrícola, v.29, p.605-613, 2009. http://dx.doi.org/10.1590/S010069162009000400010
Dacanal, G. C.; Menegalli, F. C. Selection of operational parameters for the production of instant soy protein isolate by pulsed agglomeration. Powder Technology, v.203, p.565-573, 2010. http:// dx.doi.org/10.1016/j.powtec.2010.06.023

Ferreira, M. F. P.; Dias, V. de O.; Oliveira, A.; Alonço, A. dos S.; Baumhardt, U. B. Uniformidade de vazão de fertilizantes por dosadores helicoidais em função do nivelamento longitudinal. Engenharia na Agricultura, v.18, p.297-304, 2010.

Garcia, A. P. Desenvolvimento de um sistema de controle eletromecânico para dosador de fertilizantes. Campinas: UNICAMP, 2007. 100p. Dissertação Mestrado

Garcia, A. P. Sistemas de controle Fuzzy para dosadores helicoidais de fertilizantes com acionamento elétrico. Campinas: UNICAMP, 2011. 96p. Tese Doutorado

Garcia, A. P.; Cappelli, N. L.; Umezu, C. K. Auger-type granular fertylizer distributor: matemathical model and dynamic simulation. Engenharia Agrícola, v.32, p.151-163, 2012. http:// dx.doi.org/10.1590/S0100-69162012000100016

Grisso, R.; Alley, M.; Thomason, W.; Holshouser, D.; Roberson, G. T. Precision farming tools - Variable-Rate Application (VRA). VCE Publication 442-505. Blacksburg: Virginia Tech, Virginia Polytechnic Institute and State University, 2011. 16p.

Lopes, A. J. O que é um algoritmo? Revista Nova Escola, v.238, sp, 2010.

Mossmann, V. L. da F.; Catelli, K. B. de M. F.; Libardi, H.; Damo, I. S. Determinação dos coeficientes de atrito estático e cinético utilizando-se a aquisição automática de dados. Revista Brasileira de Ensino de Física, v.24, p.146-149, 2002. http://dx.doi. org/10.1590/S1806-11172002000200010

Olieslagers, R.; Ramon, H.; Baerdemaeker, J. de. Calculation of fertilizer distribution patterns from a spinning disc spreader by means of a simulation model. Journal of Agricultural Engineering Research, v.63, p.137-152, 1996. http://dx.doi.org/10.1006/ jaer.1996.0016

Owen, P. J.; Cleary, P. W. Prediction of screw conveyor performance using the Discrete Element Method (DEM). Powder Technology, v.193, p.274-288, 2009. http://dx.doi.org/10.1016/j.powtec.2009.03.012 
Rodrigues, M. A.; Iemma, A. F. Planejamento de experimentos e otimização de processos. 2.ed. Campinas: Cárita, 2009. 358p.

Rossato, F. P.; Machado, O. D. da C.; Alonço, A. dos S.; Franck, C. J.; Francetto, T. R. Rendimento potencializado. Cultivar Máquinas, v.122, p.10-13, 2012.

Shimizu, Y.; Cundall, P. A. Three-dimensional DEM simulation of bulk handling by screw conveyors. Journal of Engineering Mechanics, v.127, p.864-872, 2001. http://dx.doi.org/10.1061/(ASCE)07339399(2001)127:9(864)
Umezu, C. K.; Cappelli, N. L. Desenvolvimento e avaliação de um controlador eletrônico para equipamentos de aplicação de insumos. Revista Brasileira de Engenharia Agrícola e Ambiental, v.10, p.225230, 2006. http://dx.doi.org/10.1590/S1415-43662006000100033

Yuan, J.; Liu, C. L.; Li, Y. M.; Zeng, Q.; Zha, X. F. Gaussian processes based bivariate control parameters optimization of variablerate granular fertilizer applicator. Computers and Electronics in Agriculture, v.70, p.33-41, 2010. http://dx.doi.org/10.1016/j. compag.2009.08.009 